\title{
Case Report on hypoplastic amelogenesis imperfecta with multiple impacted teeth
}

\author{
Dr Yogesh Chhaparwal ${ }^{1}$, Apurv Jhawar ${ }^{2}$, Dr Aditi Lele ${ }^{3}$, Dr Shubha Rathi ${ }^{4}$ \\ ${ }^{1}$ (Reader, Department of Oral Medicine and Radiology, Manipal College of Dental Sciences, Manipal \\ University, India) \\ ${ }^{2}$ (Intern, Manipal College of Dental Sciences, Manipal University, India) \\ ${ }_{3}^{3}$ (Former PG, Department of Oral Medicine and Radiology, Manipal College of Dental Sciences, Manipal \\ University, India) \\ ${ }^{4}$ (Post Graduate student, Department of Conservative Dentistry \&Endodontics,Manipal College of Dental \\ Sciences, Manipal University, India)
}

\begin{abstract}
Amelogenesis imperfecta (AI) is a hereditary disorder expressing a group of conditions that cause developmental alterations in the structure of enamel. The enamel may be hypoplastic, hypomineralized or both and affected teeth may be esthetically and functionally compromised. It has been associated with various dental anomalies, including Taurodontism, congenitally missing teeth, delayed eruption, crown resorption, and abnormal enamel thickness. Here we report a case of unusual and rare association of hypoplastic Amelogenesis Imperfecta with multiple impacted teeth. This clinical report also reviews a holisticmultidisciplinary treatment approach for the oral rehabilitation of patient with hypoplastic Amelogenesis Imperfecta with multiple impacted teeth.
\end{abstract}

Keywords: Amelogenesis Imperfecta, Hypoplastic , Full mouth rehabilitation, Impacted teeth.

\section{Introduction}

Amelogenesis imperfecta has been described as a complex group of inherited conditions that disturbs the developing enamel structure and exists independent of any related systemic disorder. AI results in poor development or complete absence of enamel of the teeth caused due to improper differentiation of Ameloblasts.This enamel anomaly may affect both the primary and permanent dentition. AI has six times more tendency than unaffected people to have impacted permanent teeth and is often associated with anomalies such as follicular cysts. ${ }^{[3]}$ Diagnosis involves exclusion of extrinsic environmental or other factors, establishment of a likely inheritance pattern, and recognition of phenotype and correlation with the dates of tooth formation to exclude a chronological developmental disturbance ${ }^{[1]}$ The enamel may be hypoplastic, hypomineralized or both, and teeth affected may be discolored, sensitive or prone to disintegration either post-eruption (post-eruptive breakdown) or pre-eruption (idiopathic resorption). ${ }^{[2]}$

\section{Case Report}

A 21 year old female patient presented with a chief complaint of multiple missing permanent teeth and discolored upper and lower teeth. There was no associated pain or difficulty in chewing reported. She resided in a non fluoridated area since her birth. Her maternal and post natal history was not significant. Family history was negative for consanguinity between her parents. No other family member had similar dental problem.General examination suggested her to be in a good state of health.Extraoral examination revealed a long and narrow face (fig. 1) with a convex profile (fig. 2). There was a macular pigmented area on the lateral aspect of her right eye. Also a few hyperpigmented spots were noticed on her right cheek and neck. Her hair and nails were normal. Intraoral examination revealed hyperpigmented gingiva with multiple unerupted permanent teeth. Teeth present were $11,54,55,21,24,25,65,31,32,36,74,75,41,84,85$, and 46 . The erupted dentition had discolored hypoplastic enamel. There was partial anodontia, few retained deciduous teeth, deep bite and a loss of vertical dimension (fig. 3-5).

Panoramic radiograph (fig. 6) showed a mixed dentition status. Radiographic examination revealed that twenty one of the permanent teeth were totally impacted $(12,13,14,15,17,18,22,23,27$, $28,33,34,35,37,38,42,43,44,45,47,48)$.Over-retention was observed in seven primary teeth $(54,55,65,74,75,84$, and 85). Permanent maxillary right and left first molars were congenitally missing (fig. 7). Pulp chambers had normal size and shape. Few impacted teeth had an increased follicular space.

A medical and dermatology consultation was sought. Her calcium levels- $9.7 \mathrm{mg} / \mathrm{mL}$, phosphorous $4.5 \mathrm{mg} / \mathrm{mL}$, alkalinephosphatase level was $127 \mathrm{IU}$ was well within the normal limits ruling out any systemic involvement. A diagnosis of linear verrucous epidermoid nevus was established for her skin pigmentation and was independent 
of the intraoral gingival hyperpigmentation. She had a normal growth and development with delayed teeth eruption.

Clinical and radiologic findings lead to a final diagnosis of amelogenesis imperfecta with multiple impacted teeth.

Treatment options for full mouth oral rehabilitation in such cases are discussed.Treatment includes replacement of missing teeth and protecting the existing dentition with crowns and bridges. Currently, the cases of AI are restored with adhesive restorative techniques, overdentures, fixed partial dentures, full porcelain crowns, porcelain fused-to metal crowns and inlay/onlay restorations constitute the contemporary treatment modalities. Overdentures have been suggested for children, because they are alterable to accommodate an active growth process. However, adequate inter occlusal space must exist. Meanwhile, full porcelain restorations are becoming increasingly popular, because of their inheriting esthetics, excellent biocompatibility and improved physical properties. Face bow transfer is done after which casts are mounted on a semi adjustable articulator. For impacted teeth lying just below the crest of the alveolar ridge, surgical exposure followed by intentional root canal treatment, cast post placement to obtain additional support for FPD. To establish loss of vertical dimension: RCT with core build up is advisable. Initially a temporary FPD (heat cured acrylic resin), which later can be replaced by a metal ceramic restoration after confirming increased vertical dimension without compromising TMJ functioning.

\section{Discussion}

AI results in poor development or complete absence of enamel of the teeth caused due to improper differentiation of ameloblasts. AI has 6 times more tendency than unaffected people to have impaction of permanent teeth and associated anomalies such as follicular cysts ${ }^{[3]}$ Impaction can be attributed to the concurrent follicular enlargement, abnormality in molecular control of eruption process, space deficiency and dental alignment problems. ${ }^{[5]}$

The primary clinical problems of AI are tooth sensitivity, loss of occlusal vertical dimension, dysfunction, and esthetics. Restoration of these defects is important not only because of esthetic and functional concerns, but also because there may be a positive psychological impact for the patient. Pitted enamel surfaces seen in AI can predispose to plaque accumulation and subsequent periodontal problems. However, orthodontic malocclusion like open contacts reduces the caries incidence. ${ }^{[5]}$ Treatment planning for patients with amelogenesis imperfecta is related to many factors: the age and socioeconomic status of the patient, the type and severity of the disorder, and the intraoral situation. An interdisciplinary approach is necessary to evaluate, diagnose, and resolve esthetic problems using a combination of periodontal, prosthodontic, and restorative treatment.

AI presents with problems of socialization, function and discomfort which may be managed by early vigorous intervention, both preventively and restoratively, with treatment continued throughout childhood and into adult life. In infancy, the primary dentition may be protected by the use of preformed metal crowns on posterior teeth and polycarbonate or composite resin crowns on anterior teeth. Restorative treatment requires long- term analysis and good oral care practice. Impacted teeth should be surgically removed and histopathological examination of all enlarged follicles should be done.

Amelogenesis Imperfecta is a syndrome in itself presenting with so many dental anomalies. Radiology plays a very important role in diagnosing various dental pathologies including assessment of enamel density to develop more appropriate treatment plan in patients with enamel defect. The dentist has to balance the decision for early intervention and long time survival of the restorations to prevent later problems.

\section{References}

[1]. Peter T, Titus S, Francis G. Full mouth rehabilitation of a case with amelogenesis imperfecta. SRM University Journal of Dental Sciences; 2010: 1(3)

[2]. Shetty YB, Shetty A. Oral Rehabilitation of a Young Adult with Amelogenesis Imperfecta: a case report. J Indian ProsthodontSoc; 2010;10(4):240-245.

[3]. Collins MA etal. Dental anomalies associated with amelogenesis imperfecta: A radiographic assessment. Oral Surg Oral Med Oral Pathol Oral Radiol Endod 1999; 88:358-64.

[4]. Canger M et al. Amelogenesis Imperfecta, Hypoplastic Type Associated with Some Dental Abnormalities: A Case Report. Braz Dent J (2010) 21(2): 170-174.

[5]. Reddy S, Nisha V, Harish BN. Hypoplastic amelogenesis imperfecta with multiple impacted teeth - report of two cases. J Clin Exp Dent. 2010; 2(4):e207-11. 


\section{Pictures:}

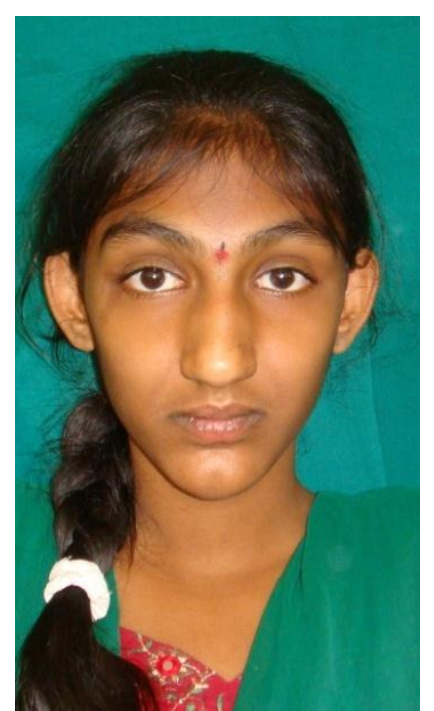

Figure 1. Front profile

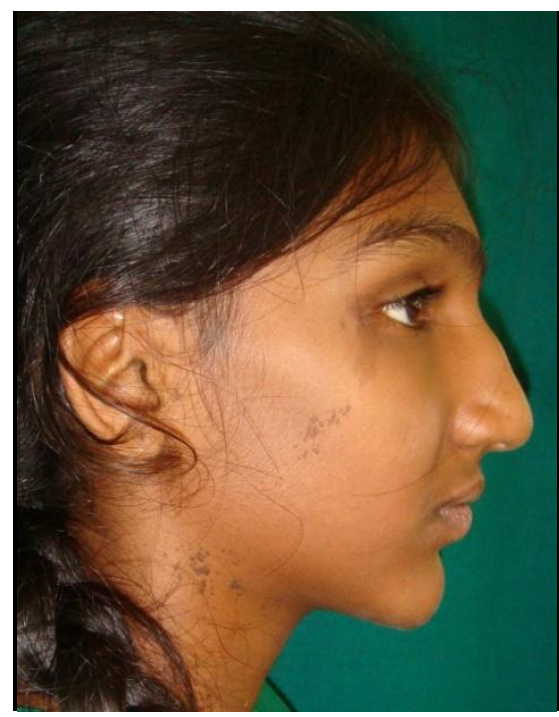

Figure 2. Side profile.

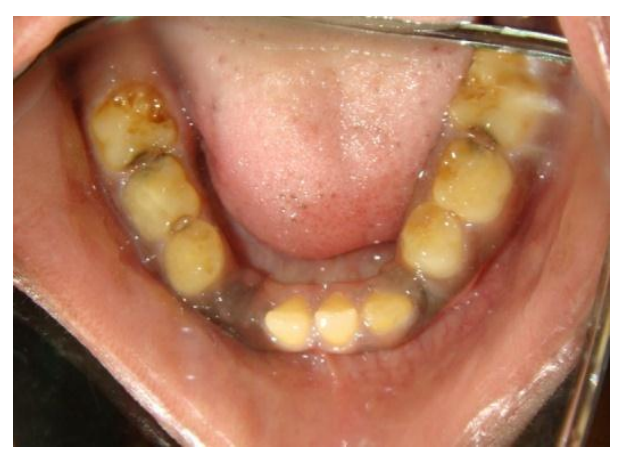

Figure 3 


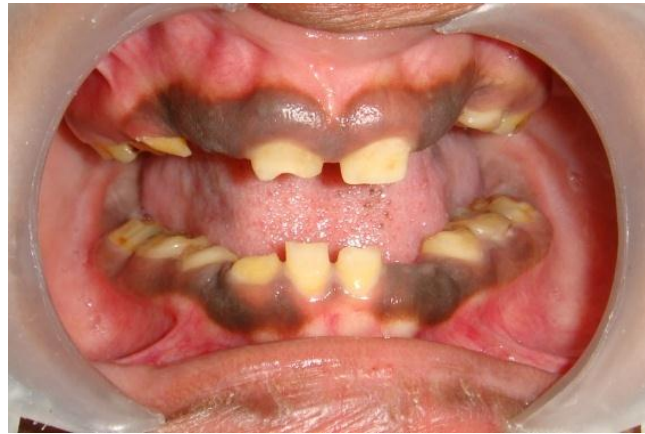

Figure 4

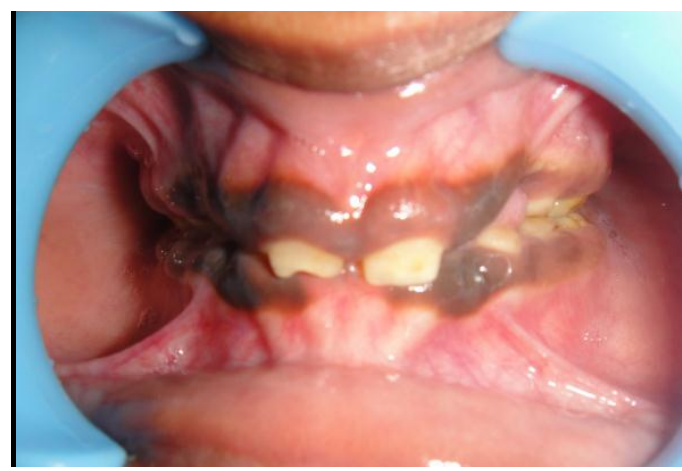

Figure 5

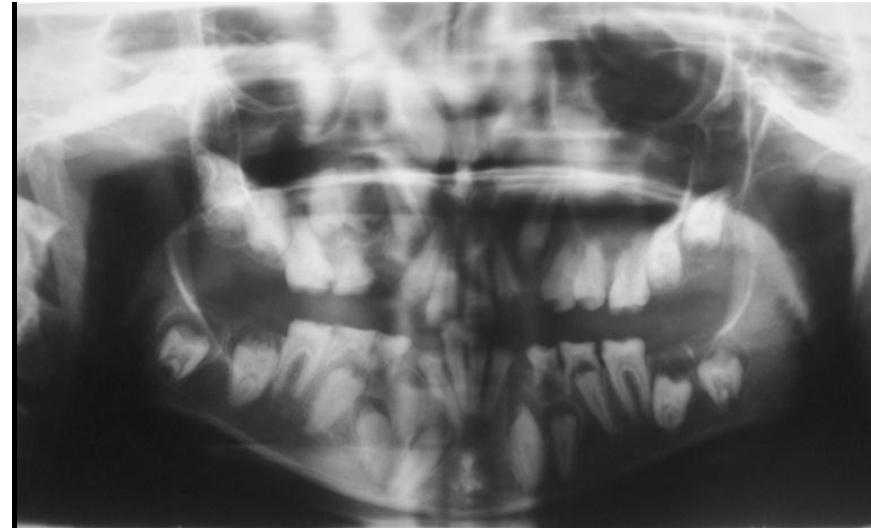

Figure 6. Panoramic Radiograph

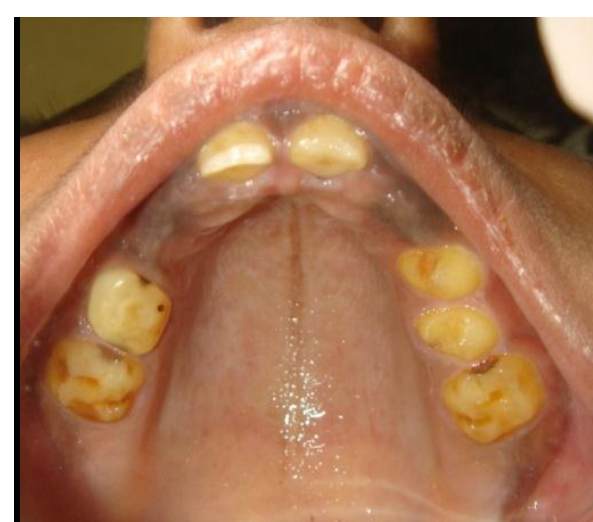

Figure 7 Missing permanent first molars 